\title{
Reply to "COVID-19 in persons with haematological cancers": a focus on myeloid neoplasms and risk factors for mortality
}

\author{
Bruno Fattizzo $\oplus^{1,2} \cdot$ Juri Alessandro Giannotta ${ }^{1,2} \cdot$ Mariarita Sciumè $^{1} \cdot$ Daniele Cattaneo $^{1} \cdot$ Cristina Bucelli $^{1}$. \\ Nicola Stefano Fracchiolla ${ }^{1}$. Francesco Onida $\mathbb{C}^{1,2} \cdot$ Luca Baldini $^{1,2} \cdot$ Wilma Barcellini $^{1} \cdot$ Alessandra lurlo $\mathbb{C}^{1}$
}

Received: 2 May 2020 / Revised: 7 May 2020 / Accepted: 13 May 2020 / Published online: 26 May 2020

(c) Springer Nature Limited 2020

We read with interest the article by $\mathrm{He}$ et al. [1] who recently reported the results of a cohort study of 128 hospitalised subjects with haematological cancers in Wuhan. Thirteen patients developed COVID-19 infection along a 3 -week period (Jan 23-Feb 13). Patients were compared with 226 health-care providers, and similar COVID-19 infection rates were found (10\% versus $7 \%)$. However, haematological patients had more severe COVID-19 and more deaths, without a correlation with haematological cancer type. Since patients with myeloid malignancies are usually elderly, immunocompromised, and generally have a higher infectious risk, particularly myelofibrosis, high-risk myelodysplastic syndromes (MDS), and acute myeloid leukaemia (AML) [2], we evaluated a series of 1250 patients with myeloid neoplasms regularly followed at a public university hospital in Milan, Italy. To the best of our knowledge, no specific data about the course of COVID-19 infection in myeloid neoplasms, have been reported so far, but those included in the study by $\mathrm{He}$ et al. (58 patients, 50 AML, and 8 MDS, of whom 5 contracted COVID-19). The latter were however all hospitalised, whilst the higher proportion of haematology care encompass outpatient clinic and day-care service; moreover, myeloproliferative neoplasms (MPN) were not included. We collected clinical and laboratory data from patients with AML, MDS, MPN, and MDS/MPN diagnosed with COVID-19 between March, 10 and April, 242020 (6 weeks period). Sixteen patients $(1.28 \%)$ turned positive (nasal-pharyngeal swab) and were included in the analysis (Table 1). Subjects were mainly males $(62.5 \%)$, all but 3 aged $>60$ years and with at least

Bruno Fattizzo

bruno.fattizzo@unimi.it

1 Fondazione IRCCS Ca' Granda Ospedale Maggiore Policlinico, Milan, Italy

2 University of Milan, Milan, Italy one comorbidity (mainly arterial hypertension and metabolic disorders, $56 \%$ and $44 \%$, respectively). Fifty percent of cases were receiving antihypertensive drugs, $37.5 \%$ renin-angiotensin-axis inhibitors. These data reflect the average age and comorbidity burden of myeloid outpatient series, that differ from hospitalised patients reported by $\mathrm{He}$ et al., were median age of subjects developing COVID-19 was 35 year (range, 23-53 years) and prevalence of cardioand cerebrovascular diseases were lower (24\%). Moreover, in our series, COVID-19 prevalence of $1.28 \%$ was higher than that observed in Lombardy in the same period $(0.5-0.7 \%)$ [3], possibly indicating higher susceptibility of myeloid outpatients versus hospitalised one [1]. Interestingly, two of our patients were health-care professionals, and might have been professionally exposed.

Haematologic diagnoses were equally distributed and $18 \%$ of patients were receiving specific therapies (i.e. two tyrosine kinase inhibitors, BCR/ABL-nilotinib, and JAKinhibitor ruxolitinib, and 1 recombinant erythropoietin), but no chemotherapies. Most patients presented with fever, cough and dyspnoea, and $80 \%$ required hospital admission. Twelve percent of cases required admission to the intensive care unit, similarly to the about $10 \%$ rate reported in Italian general population [3]. Fifty percent showed bilateral pneumonia, whilst only $25 \%$ displayed the classical interstitial pattern. Contrarily, $\mathrm{He}$ et al. found similar lung typical changes in patients with or without haematologic malignancies, although this might be partly due to systematic CT scan evaluation (easier to access for hospitalised patients). The atypical radiologic presentation in our series [4], may be due to a mitigating effect of the underlying haematologic disease on the degree of immune system activation. On the other hand, this insidious presentation may delay diagnosis, particularly in case of negative swab. Whether some of the haematological therapies, many of which exert antiinflammatory/immunomodulatory activities, constitute a favourable or detrimental factor would need future ad hoc studies. Of note, JAK-inhibitors 
Table 1 Clinical features of myeloid patients with COVID-19 infection.

\begin{tabular}{|c|c|c|c|c|}
\hline & All $(n=16)$ & Alive $(n=11)$ & Dead $(n=5)$ & $p$ value \\
\hline \multicolumn{5}{|l|}{ Clinical features at baseline } \\
\hline Median age, years (range) & 77 (27-94) & $77(47-89)$ & $85(64-94)$ & 0.15 \\
\hline $\mathrm{M} / \mathrm{F}$ & $10 / 6$ & $5 / 6$ & $5 / 0$ & 0.04 \\
\hline LR-MDS, $n(\%)$ & $4(25)$ & $3(28)$ & $1(20)$ & 0.59 \\
\hline MPN, $n(\%)$ & $5(31)$ & $4(36.4)$ & $1(20)$ & 0.59 \\
\hline MDS/MPN, $n(\%)$ & $3(19)$ & $2(18)$ & $1(20)$ & 0.69 \\
\hline HR-MDS/AML, $n(\%)$ & $4(25)$ & $2(18)$ & $2(40)$ & 0.4 \\
\hline \multicolumn{5}{|l|}{ Comorbidities } \\
\hline CCI, median (range) & $1(0-6)$ & $0(0-4)$ & $3(1-6)$ & 0.03 \\
\hline Hypertension, $n(\%)$ & $9(56)$ & $5(45)$ & $4(80)$ & 0.18 \\
\hline Other cardiovascular, $n(\%)$ & $6(37)$ & $4(36)$ & $2(40)$ & 0.56 \\
\hline Autoimmune, $n(\%)$ & $2(12.5)$ & $2(18)$ & $0(0)$ & 0.42 \\
\hline Thrombosis, $n(\%)$ & $5(31)$ & $3(27)$ & $2(40)$ & 0.4 \\
\hline Metabolic, $n(\%)$ & $7(44)$ & $3(27)$ & $4(80)$ & 0.1 \\
\hline Neurologic, $n(\%)$ & $3(19)$ & $2(18)$ & $1(20)$ & 0.75 \\
\hline Nephrologic, $n(\%)$ & $2(12.5)$ & $0(0)$ & $2(40)$ & 0.09 \\
\hline Respiratory, $n(\%)$ & $1(6)$ & $1(9)$ & $0(0)$ & 0.66 \\
\hline Solid tumour, $n(\%)$ & $3(19)$ & $2(18)$ & $1(20)$ & 0.75 \\
\hline Hepatic, $n(\%)$ & $1(6)$ & $0(0)$ & $1(20)$ & 0.33 \\
\hline \multicolumn{5}{|l|}{ Haematologic treatment } \\
\hline Follow up & $5(31)$ & $5(45)$ & $0(0)$ & 0.08 \\
\hline TKI & $2(12.5)$ & $1(9)$ & $1(20)$ & 0.57 \\
\hline BSC & $5(31)$ & $1(9)$ & $4(80)$ & 0.003 \\
\hline Steroids & $3(19)$ & $3(27)$ & $0(0)$ & 0.5 \\
\hline ESA & $1(6)$ & $1(9)$ & $0(0)$ & 0.66 \\
\hline \multicolumn{5}{|l|}{ Other medications } \\
\hline Anti-hypertensive, $n(\%)$ & $8(50)$ & $5(45)$ & $3(60)$ & 0.42 \\
\hline ACEi/ARBs, $n(\%)$ & $3(19)$ & $2(18)$ & $1(20)$ & 0.75 \\
\hline Anti-coagulant, $n(\%)$ & $2(12.5)$ & $1(9)$ & $1(20)$ & 0.57 \\
\hline \multicolumn{5}{|c|}{ Haematologic parameters before COVID-19 infection } \\
\hline Median $\mathrm{Hb}, \mathrm{g} / \mathrm{dL}$ (range) & $10.2(6.3-14.7)$ & $10.3(7.8-14.7)$ & $8.2(6.3-12)$ & 0.15 \\
\hline Median ANC, $\times 10^{9} / \mathrm{L}$ (range) & $3.4(0.12-16.6)$ & $2.93(1.5-6.6)$ & $7.7(0.12-16.6)$ & 0.06 \\
\hline Median ALC, $\times 10^{9} / \mathrm{L}$ (range) & $1.2(0.4-4.0)$ & $1.8(0.4-4.0)$ & $1.0(0.68-2.8)$ & 0.25 \\
\hline Median PLT, $\times 10^{9} / \mathrm{L}$ (range) & $166(6-398)$ & $166(13-356)$ & $107(6-398)$ & 0.8 \\
\hline \multicolumn{5}{|l|}{ Clinical features at COVID-19 infection } \\
\hline \multicolumn{5}{|l|}{ Symptoms at onset } \\
\hline Fever, $n(\%)$ & $9(56)$ & $6(55)$ & $3(60)$ & 0.57 \\
\hline Cough, $n(\%)$ & $6(37.5)$ & $5(45)$ & $1(20)$ & 0.43 \\
\hline Dyspnoea, $n(\%)$ & $5(31)$ & $4(36)$ & $1(20)$ & 0.59 \\
\hline Diarrhoea, $n(\%)$ & $2(12.5)$ & $2(18)$ & $0(0)$ & 0.66 \\
\hline Fatigue, $n(\%)$ & $1(6)$ & $1(9)$ & $0(0)$ & 0.66 \\
\hline Asymptomatic, $n(\%)$ & $2(12.5)$ & $1(9)$ & $1(20)$ & 0.57 \\
\hline Radiologic findings & $n=12$ & & & \\
\hline Unremarkable, $n(\%)$ & $1(8)$ & $0(0)$ & $1(20)$ & 0.33 \\
\hline Monolateral focal lesions, $n(\%)$ & $2(17)$ & $0(0)$ & $2(40)$ & 0.09 \\
\hline Bilateral focal lesions, $n(\%)$ & $6(50)$ & $5(40)$ & $1(20)$ & 0.29 \\
\hline Interstitial pneumonia, $n(\%)$ & $3(25)$ & $1(10)$ & $2(40)$ & 0.24 \\
\hline Associated pleural effusion, $n(\%)$ & $1(8)$ & $0(0)$ & $1(20)$ & 0.33 \\
\hline
\end{tabular}


Table 1 (continued)

\begin{tabular}{|c|c|c|c|c|}
\hline & All $(n=16)$ & Alive $(n=11)$ & Dead $(n=5)$ & $p$ value \\
\hline \multicolumn{5}{|l|}{ Level of care } \\
\hline Hospital admission, $n(\%)$ & $13(80)$ & $9(82)$ & $4(80)$ & 0.73 \\
\hline ICU admission, $n(\%)$ & $2(12,5)$ & $1(10)$ & $1(20)$ & 0.57 \\
\hline Home quarantine, $n(\%)$ & $3(19)$ & $2(20)$ & $1(20)$ & 0.75 \\
\hline \multicolumn{5}{|l|}{ Treatments } \\
\hline Oxygen, $n(\%)$ & $12(75)$ & $7(64)$ & $5(100)$ & 0.15 \\
\hline NIV (CPAP included), $n(\%)$ & $2(12.5)$ & $1(9)$ & $1(20)$ & 0.57 \\
\hline Steroids, $n(\%)$ & $5(31)$ & $3(27)$ & $2(40)$ & 0.56 \\
\hline Heparin, $n(\%)$ & $5(31)$ & $3(27)$ & $2(40)$ & 0.56 \\
\hline $\mathrm{HCQ}, n(\%)$ & $9(56)$ & $6(55)$ & $3(60)$ & 0.57 \\
\hline Antiviral, $n(\%)$ & $3(19)$ & $3(27)$ & $0(0)$ & 0.42 \\
\hline Antibiotics, $n(\%)$ & $9(56)$ & $6(55)$ & $3(60)$ & 0.7 \\
\hline Anti-IL6, $n(\%)$ & $2(12.5)$ & $2(18)$ & $0(0)$ & 0.66 \\
\hline
\end{tabular}

All patients showed a positive nasopharyngeal swab.

$M D S$ myelodysplastic syndrome, $M D S / M P N$ myelodysplastic/myeloproliferative syndrome, $A M L$ acute myelogenous leukaemia, $C C I$ Charlson Comorbidity Index, ESA erythropoiesis stimulating agent, TKI tyrosine kinase inhibitos, $B S C$ best supportive care, $A C E i$ angiotensin converting enzyme inhibitor, $A R B s$ angiotensin receptor blocker, $H b$ haemoglobin, $A N C$ absolute neutrophil count, $A L C$ absolute lymphocyte count, $P L T$ platelets, $I C U$ intensive care unit, NIV non-invasive ventilation, $C P A P$ continuous positive airway pressure, $H C Q$ hydroxychloroquine.

Bold values indicate $p$ values that are borderline for statistical significance and may suggest clinical meaningful differences.

used in myeloproliferative neoplasms are under investigation in COVID-19 infection, aimed at downregulating the cytokine storm $[5,6]$.

Regarding COVID-19 therapies, all but four patients received oxygen support, together with hydroxychloroquine $(56 \%)$, steroids $(30 \%)$, and heparin $(30 \%)$. About $1 / 5$ were treated with antiviral agents, and only 2 with IL1/IL6 inhibitors. Interestingly, $56 \%$ of subjects also received intravenous antibiotics because of likely superimposed bacterial pneumonia. This finding was similar to what reported by $\mathrm{He}$ et al., who found a high prevalence of bacterial, fungal, and virus co-infections in patients with haematologic cancers compared with controls.

Figure 1 shows laboratory parameters at COVID-19 diagnosis and at the last follow up: almost all patients presented with lymphopenia, increased C-reactive protein (CRP), D-dimer, and prolonged prothrombin time. Similarly, $\mathrm{He}$ et al. reported that COVID-19 positive subjects with haematological cancer had significantly higher levels of CRP and procalcitonin, lower haemoglobin, lymphocyte, and platelet concentrations compared with the cohort without haematological cancer, possibly due to the effect of haematologic therapies. Since our patients were all outpatients and none was on chemotherapy, our results are more likely attributable to COVID-19 infection itself.

Five patients died, all older than 60 years, males $(p=0.04)$ and with a higher Charlson comorbidity index $(p=0.03)$. These risk factors, already reported for the general population, are apparently more detrimental than underlying haematologic disease. In fact, fatal outcome was not associated with a specific myeloid disease or its therapy. Regarding laboratory parameters, patients with fatal outcome showed significantly lower $\mathrm{Hb}$ levels and lymphocyte counts, and increased neutrophils, CRP, D-dimer, and PT ratio, as compared with survivors (Fig. 1). Regarding the latter two, we did not find clinical evidence of thrombo-haemorrhagic complications in our series. The association of fatality with lymphopenia and increased neutrophils has already been reported for the general population [7], whilst the severity of anaemia as predictor of mortality is a novel finding, and suggests an important contribution to the hypoxic state. On the whole, all these factors accounted for a high mortality rate of $30 \%$, similar to that reported for non-haematologic ICU admitted patients [8]. In the study by $\mathrm{He}$ et al., fatality rate was even higher among onco-haematologic patients $(60 \%)$, possibly due to the higher illness burden in hospitalised cases and related with increased baseline D-dimer levels.

In conclusion, patients with myeloid neoplasms contracting COVID-19 infection may have atypical insidious presentation and suffer from higher mortality. The latter, although mainly related to age and comorbidities, may be predicted by anaemia, lymphopenia, increased inflammatory markers, prolonged PT, and elevated D-dimer. Superimposed bacterial infections seem to play an important role in this population. Finally, since risk for COVID-19 contagion in myeloid patients may be higher, the maximal level of surveillance and protective isolation is warranted. 

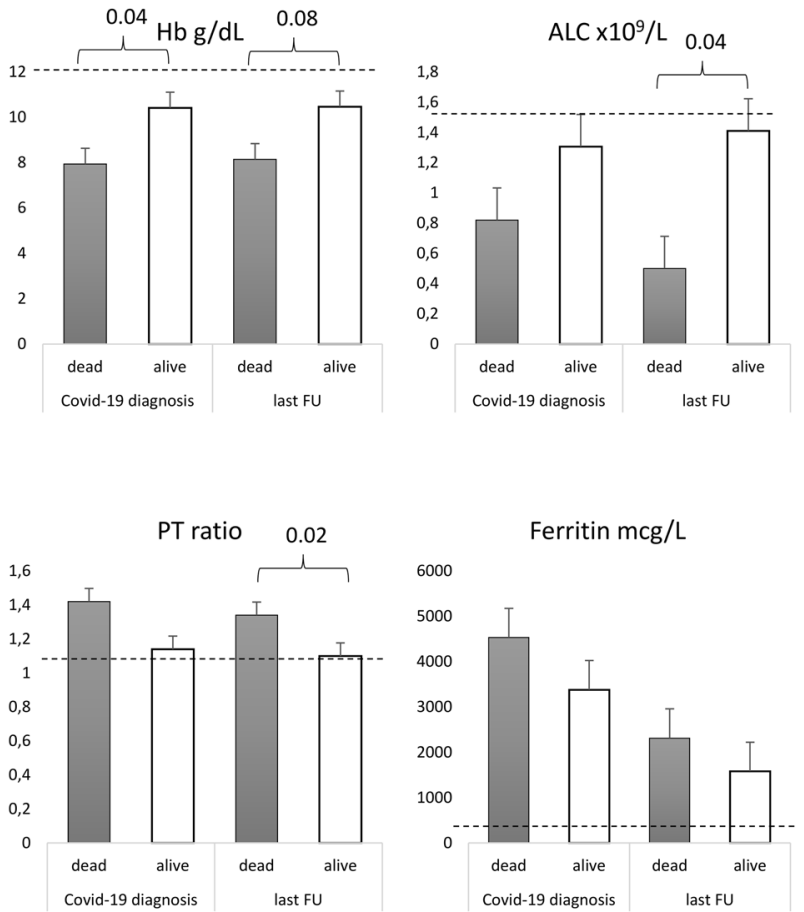

Fig. 1 Laboratory parameters of myeloid patients diagnosed with COVID-19 infection. Data are presented as mean \pm SE both at diagnosis of COVID-19 infection and at the last follow up. Horizontal dashed lines represent lower limit of normality for $\mathrm{Hb}$, ALC, and PLT,

Author contributions All authors followed patients, collected data, wrote the article and revised it for important intellectual content.

\section{Compliance with ethical standards}

Conflict of interest The authors declare that they have no conflict of interest.

Publisher's note Springer Nature remains neutral with regard to jurisdictional claims in published maps and institutional affiliations.

\section{References}

1. He W, Chen L, Chen L, Yuan G, Fang Y, Chen W, et al. COVID19 in persons with haematological cancers. Leukemia. 2020. https://doi.org/10.1038/s41375-020-0836-7.

2. Popescu CM, Ursache AL, Feketea G, Bocsan C, Jimbu L, Mesaros $\mathrm{O}$, et al. Are community acquired respiratory viral infections an underestimated burden in hematology patients? Microorganisms. 2019;7:E521.

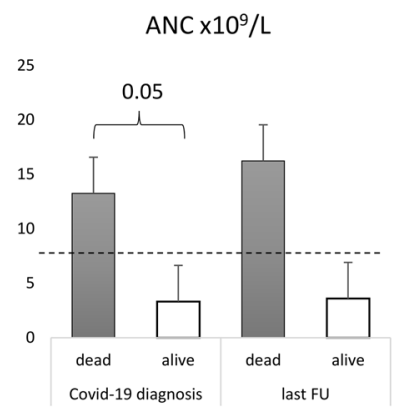

PLT $\times 10^{\%} / L$
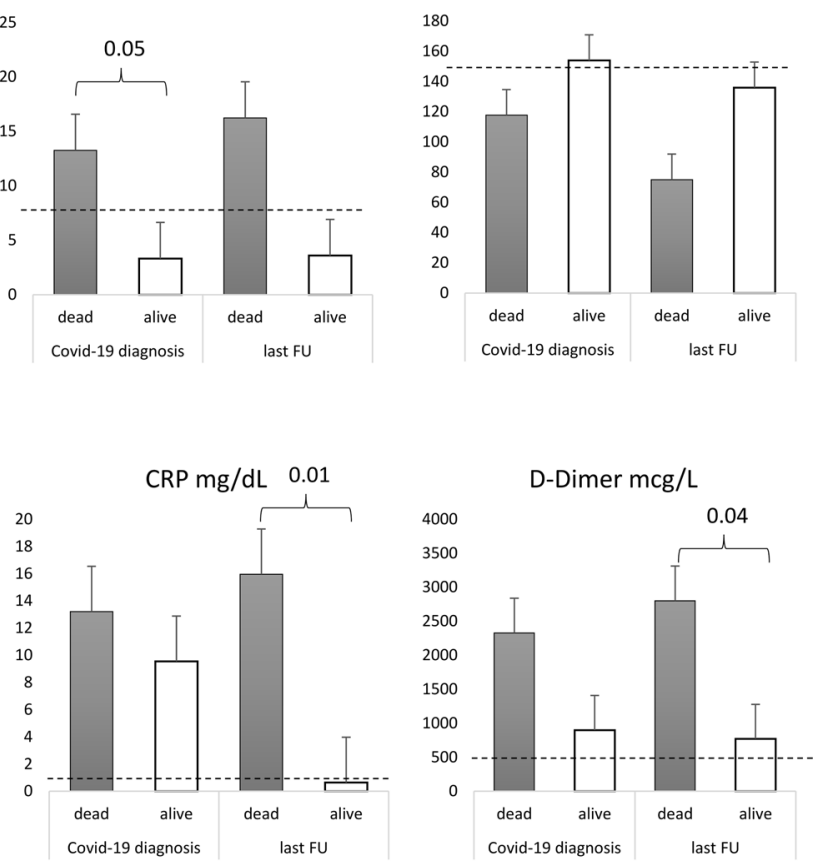

and upper limit of normality for ANC, PT, Ferritin, CRP, and D-dimer. $\mathrm{Hb}$ haemoglobin, Plt platelets, ANC absolute neutrophil counts, ALC absolute lymphocyte counts, PT prothrombin time, CRP C-reactive protein.

3. COVID-19 contagions trends in Italy. Updated April, 24, 2020. Accessed 24 April 2020. https://aifi.net/andamento-contagi-covid19-coronavirus-per-regione.

4. Kooraki S, Hosseiny M, Myers L, Gholamrezanezhad A. Coronavirus (COVID-19) outbreak: what the department of radiology should know. J Am Coll Radio. 2020;17:447-51. 121 https://doi.org/10. 1016/j.jacr.2020.02.008.

5. Sarzi-Puttini P, Giorgi V, Sirotti S, Marotto D, Ardizzone S, Rizzardini G, Antinori S, Galli M. COVID-19, cytokines and immunosuppression: what can we learn from severe acute respiratory syndrome? Clin Exp Rheumatol. 2020;38: 337-42.

6. Iurlo A, Cattaneo D, Bucelli C. Management of Myelofibrosis: from diagnosis to new target therapies. Curr Treat Options Oncol. 2020;21:46.

7. Frater JL, Zini G, d'Onofrio G, Rogers HJ COVID-19 and the clinical hematology laboratory. Int J Lab Hematol. 2020. https:// doi.org/10.1111/ijlh.13229.

8. Grasselli G, Zangrillo A, Zanella A, Antonelli M, Cabrini L, Castelli A, et al. COVID-19 Lombardy ICU Network. Baseline characteristics and outcomes of 1591 patients infected with SARSCoV-2 admitted to ICUs of the Lombardy Region, Italy. JAMA. 2020. https://doi.org/10.1001/jama.2020.5394. 